\title{
Resolving the atomistic structure and morphology of functional nanomaterials
}

\author{
Katharine Lynn Page ${ }^{1}$, Jue Liu ${ }^{1}$
}

${ }^{1}$ Chemical And Engineering Materials Division, Oak Ridge National Laboratory, Oak Ridge, United States E-mail: pagekl@ornl.gov

It is widely recognized that the internal and surface atomistic structure, composition, and morphology (size and shape) of nanomaterials can all strongly influence physical and chemical properties, yet many characterization techniques focus on one aspect or length-scale regime exclusively when developing quantitative nanostructure models. In this contribution we demonstrate the use of differential evolution refinement based on Debye scattering equation calculations for combined analysis of x-ray and neutron pair distribution function, diffraction, and small angle scattering data towards unraveling intricate structure-property relationships in functional nanomaterial families. For example, we quantify the facet-specified photocatalytic hydrogen evolution activity of a family of anatase TiO2 nanocrystals with different $\{001\}$ to $\{101\}$ ratios, indicating that intermediate levels of the two facets show optimal photocatalytic activity. We investigate the local and longrange structure of synthetic layered $\delta-\mathrm{MnO} 2$ (Birnessite or Chalcophanite), from formation through dehyration. Data-based models with turbostratic stacking disorder and interlayer/surface chemistry reveal that interlayer water molecules and hydrogen bonding play key roles in maintaining the long range stacking coherence in the c-axis (or stacking) direction, having important implications for terrestrial $\mathrm{Mn}$ recycling and water oxidation catalysis. Overall, we demonstrate how simultaneous structure and morphology quantification techniques can be broadly applied to improve our understanding of technologically important nanomaterials, while presenting some bounds to that corollary through experiment and simulation.

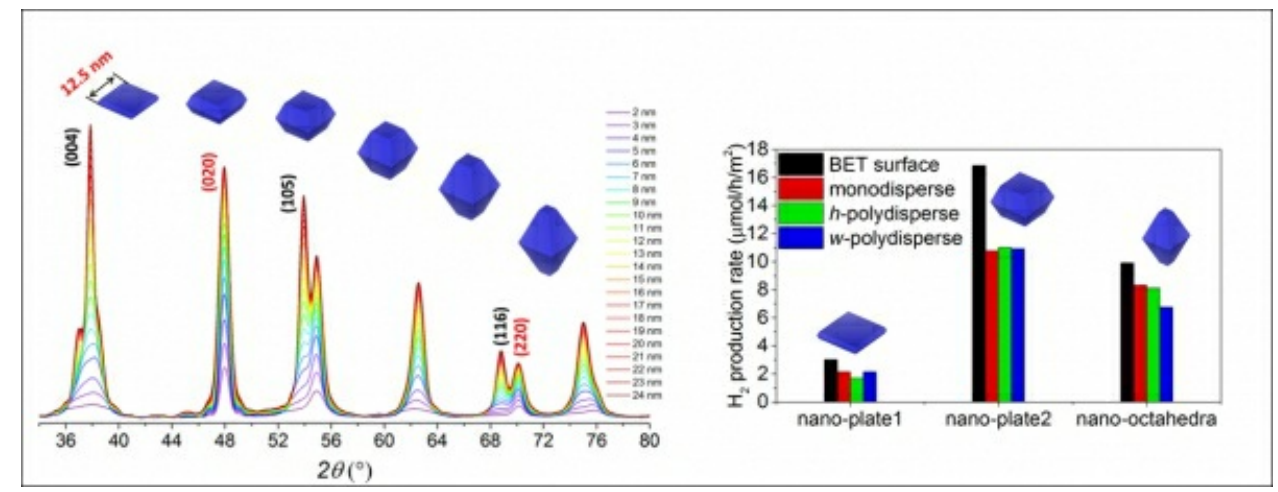

Keywords: pair distribution function, Debye scattering equation, nanostructure 